\title{
Analysis of a multi-wavelength time-resolved observation of a coronal loop
}

\author{
F. Reale ${ }^{1}$ and A. Ciaravella ${ }^{2}$ \\ 1 Dipartimento di Scienze Fisiche \& Astronomiche, Sezione di Astronomia, Università di Palermo, Piazza del Parlamento 1, \\ 90134 Palermo, Italy \\ e-mail: reale@astropa.unipa.it \\ 2 INAF - Osservatorio Astronomico di Palermo “Giuseppe S. Vaiana”, Piazza del Parlamento 1, 90134 Palermo, Italy \\ e-mail: ciarave@astropa.unipa.it
}

Received 6 October 2005 / Accepted 5 December 2005

\begin{abstract}
Aims. We analyze a well-defined loop system detected in a time-resolved observation in several spectral bands to characterize the structure and evolution of coronal loops.

Methods. The dataset includes simultaneous sequences of images in the $171 \AA$, $195 \AA$ and $284 \AA$ filter bands of TRACE, and in one filter of Yohkoh/SXT, with a time coverage of about $2.5 \mathrm{~h}$, and two rasters taken with SoHO/CDS in twelve relevant lines, forming between log $T \approx 5.4$ (O V $629 \AA$ ) and $\log T \approx 6.4$ (Fe XVI $360 \AA$ ). The loop is initially best seen in the TRACE $195 \AA$ filter band, with some correspondence to the simultaneous SXT images, and later in the $171 \AA$ filter band, with good correspondence to the CDS raster images in the lines with formation temperature around $\log T \approx 6.0-6.1$. We have taken as pixel-by-pixel background the latest TRACE, Yohkoh and CDS images where the loop has faded out. We examine the loop morphology evolution, the light curves, the TRACE filter ratio distribution and evolution, the images and emission measure from the CDS spectral lines.

Results. Our analysis detects that, after background subtraction, the emission along the loop and its evolution are non-uniform, especially in the $171 \AA$ filter band, and that the TRACE 195/171 filter ratio has a moderately non-uniform distribution along the loop and evolves over time. Both the light curves and the filter ratio evolution indicate a globally cooling loop. Relatively hot plasma may be present at the beginning while, during the first CDS raster, the data indicate a rather moderate thermal structuring of the loop.

Conclusions. Our analysis supports a coherent scenario across the different bands and instruments, points out difficulties in diagnostic methods and establishes quantitative basis for detailed forward modeling.
\end{abstract}

Key words. Sun: UV radiation - Sun: corona - Sun: X-rays, gamma rays

\section{Introduction}

As pointed out in the first X-ray images at high resolution (e.g. Vaiana et al. 1973), coronal loops are the building blocks of the X-ray luminous solar corona. Hot coronal loops are known to be steady and stable over time scales longer than the characteristic plasma cooling times, and equilibrium scaling laws ruling some physical conditions (i.e. the maximum temperature, the pressure and the heating rate) generally hold (Rosner et al. 1978).

The high space and time resolution of the Transition Region And Coronal Explorer (TRACE, Handy et al. 1999) telescope has brought new insight into the structure of the solar corona, and allowed investigators to address new issues driven by the detection, for instance, of the filamentary structure of the coronal loops (Schrijver et al. 1999; Reale \& Peres 2000) and of oscillations and propagating waves (e.g. De Moortel et al. 2000; Nakariakov \& Ofman 2001).
In parallel to such achievements, the interpretation of "conventional" loop structures as observed with TRACE is under debate. The first observations with TRACE detected steady loops with a ratio of the fluxes in the $195 \AA$ and $171 \AA$ filter passbands almost constant along the loop (Lenz et al. 1999). The ratio of the emission in different passband filters is typically used as a temperature indicator. Unfortunately the temperature diagnostics using TRACE filters are particularly difficult, because the functions linking the ratios to the temperature are multi-valued. Nevertheless, if one assumes that most of the emission comes from plasma in the temperature range of maximum filter sensitivity, the function can be inverted and what is typically obtained for TRACE loops is that they are almost isothermal, much more than predicted by standard static loop models, and also overdense with respect to static loops at $\sim 1 \mathrm{MK}$. Is this a new class of loops? Soon after, it was shown that an alternative interpretation is possible: bundles of thin strands, each behaving as a single "standard" loop, 
convolved with the TRACE temperature response could appear as a single almost isothermal loop (Reale \& Peres 2000). Another possibility has been invoked that long loops are mostly isothermal if heated at their footpoints (Aschwanden et al. 2001). This model suffers from the problem that footpointheated loops have been proven to be thermally unstable (e.g. Serio et al. 1981) and therefore cannot be long-lived, as is observed. A further alternative is to explain observations by steady non-static loops, i.e. with significant flows inside (Winebarger et al. 2001, 2002). This hypothesis also does not seem to address this issue (Patsourakos \& Klimchuk 2004). One of the reasons why the situation is so unclear is inherent in the data: although sometimes bright and well defined, the loops under analysis are always surrounded by other bright structures, which often intersect them along the line of sight. Moreover, a uniform diffuse background emission also affects the temperature diagnostics, by adding systematic offsets which alter the filter ratio values. This problem emerged dramatically when the analysis of the same loop structure observed with the Soft X-ray Telescope (SXT, Tsuneta et al. 1991) on board Yohkoh (Ogawara et al. 1991) led to three different results depending on the different ways treating the background (Priest et al. 2000; Aschwanden 2001; Reale 2002). One obvious way to check the validity of the data analysis and of the loop diagnostics is to compare data from imaging instruments to simultaneous and cospatial data obtained from spectroheliographic instruments like the Coronal Diagnostic Spectrometer (CDS, Harrison et al. 1995) on board SoHO (Domingo et al. 1995). A loop observed on the solar limb with SoHO/CDS was analyzed by Schmelz et al. (2001), who found that whereas single line ratios tend to yield flat temperature distributions along the loop, a careful reconstruction of the emission measure distribution vs temperature (DEM) at selected points along the loop shows that this may not be a realistic result. A whole series of works started from this study reconsidering and questioning the basic validity of the temperature diagnostics with TRACE and emphasizing once again the importance of the background subtraction, but also the need to obtain accurate spectral data (Schmelz 2002; Martens et al. 2002; Aschwanden 2002; Schmelz et al. 2003). Similar results but different conclusions were reached by Landi \& Landini (2004), and Landi \& Feldman (2004) who analyzed a loop observed with SoHO and, finding it nearly isothermal, consider this evidence as real and invoke a non-constant cross-section to explain it. From their analysis of SoHO/CDS data compared to other similar analyses made by other authors, Schmelz et al. (2005) proposed that there may be two different classes of loops, multi-thermal and isothermal, while Aschwanden \& Nightingale (2005) analyzed the thinnest loop structures detected with TRACE and found that a few are isothermal along the line of sight and may therefore be elementary loop components.

Another puzzling issue, certainly linked to the loop isothermal appearance, is the loop overdensity. In order to explain both these pieces of evidence, several authors claim that the loop cannot be at equilibrium and it must be filamented and cooling from a hotter state, probably continuously subject to heating episodes (nanoflares, Warren et al. 2002; Warren et al. 2003; Cargill \& Klimchuk 2004). The presence of nanoflares might explain the presence of coronal loops, stable although heated at the footpoints and with a peaked distribution of emission measure, as observed in active stars (Testa et al. 2005). The analysis of simultaneous Yohkoh and TRACE data has shown an anticoincidence between hot and cooler loops (Nagata et al. 2003; Schmieder et al. 2004), and however, a moderate but finite width of the DEM loops.

Time-dependent modeling of one coronal loop observed with TRACE showed out that the detailed description of the evolution of this loop requires a heating located at intermediate position between the apex and the footpoints, probably initially high and then slowly decaying (Reale et al. 2000).

The current debate in the interpretation of coronal loop observations points out the presence of intrinsic limitations in the information that one can derive from present-day data. Here, we take the analysis of a multi-wavelength observation of a time-evolving coronal loop as a guide to study how deep one can go in the diagnostics and characterization of the loop, and sets the basis for further analysis through detailed forward modeling which we leave for a future work. To this purpose we have searched for the observation of a loop under particularly good conditions for analysis: a simple and welldefined system, as isolated as possible, imaged in more than one TRACE filter band, in several SoHO/CDS spectral lines and with Yohkoh/SXT, and for a time period of more than one hour. Its disappearance allows us to use the last images as a point-to-point background to be subtracted. We try to use the coherence of the structure, its evolution and the spectral data to extract the maximum possible information from the data.

The selection of the loop observation, its description and the methods of the data analysis are illustrated in Sect. 2, the results of the analysis are shown in Sect. 3 and they are discussed in Sect. 4; we draw our conclusion in Sect. 5.

\section{The observation and data analysis}

\subsection{The observation campaign}

From the list of SoHO campaigns available on the Web site http://sohodata.nascom.nasa.gov/cgi-bin/gui, we have selected the campaign, with ID number 5170, named Active Region Study. The observation date is 13 May 1998 and the coordinator is Robert Walsh. The campaign includes data from TRACE, SoHO/CDS and Yohkoh/SXT. TRACE data consist of a $3.5 \mathrm{~h}$ time-sequence of $1024 \times 1024$ full resolution images in all three filters (171 $\AA, 195 \AA, 284 \AA)$. Yohkoh/SXT data consist of a time-sequence of half-resolution ( $5^{\prime \prime}$ pixel size) full-disk images mostly in a single filter, with a good time overlap with the time period of the TRACE data. SoHO/CDS data include several rasters but only two of them with good spectral resolution and a relevant field of view (see Sect. 2.5). The first raster overlaps the TRACE observation. Figure 1 shows the time coverage of each piece of observation relevant for the present analysis. The TRACE frames span from 6:30 UT to 10 UT with two significant gaps of about $20 \mathrm{~min}$ around 7:15 UT and 8:50 UT, which divide the TRACE data into three main parts. Most of the loop evolution is included in the first two TRACE segments. These are 


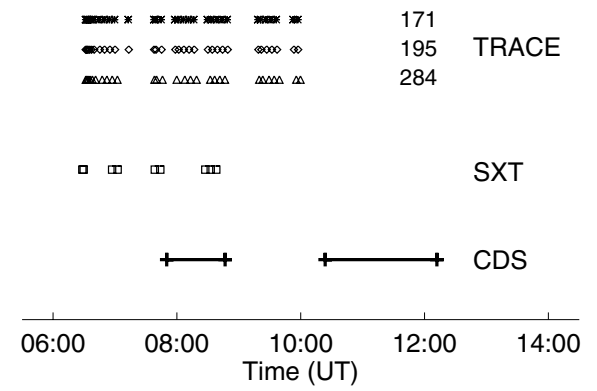

Fig. 1. Time coverage of the relevant observations of the loop selected for the present analysis. The times of the TRACE and Yohkoh frames are marked with data points (stars, diamonds triangles, and squares for the TRACE $171 \AA, 195 \AA$ and $284 \AA$ filters and for SXT, respectively), the time lapses of the two relevant CDS rasters are marked with the segments.

well covered by the SXT data (until 8:50 UT). The first CDS raster occurs during the second TRACE segment. The second (longer) raster is taken after the end of the TRACE data. For completeness, in order to report on the loop evolution at times before the campaign, we have partially analyzed TRACE data in the $171 \AA$ and $195 \AA$ filter bands (about 50 additional images in each filter) taken between 0:18 UT and 6:20 UT and one Yohkoh/SXT image taken at 5:26 UT.

\subsection{The loop selection}

The loop has been selected on the TRACE images. It appears as an entire loop in several $171 \AA$ filter images, and it is clearly visible also in the $195 \AA$ band. A mostly visible loop is able to provide complete constraints on diagnostics and models. Many loops are faint around their apex, because of the stratification due to gravity. The loop is bright, i.e. observed with good count statistics and with a high contrast over the background. We have searched for a loop as far as possible free from other structures intersecting the line of sight, which might be difficult to disentangle from the analyzed loop. The right half of the loop appears to match well this requirement. The loop is $\approx 10^{10} \mathrm{~cm}$ long and lies on the solar disk $\left(X_{\text {sol }}=+230^{\prime \prime}, Y_{\text {sol }}=+470^{\prime \prime}\right)$ (see also Fig. 2). We prefer a loop located on the disk, because, on the limb, more coronal structures are met along the line of sight (as suggested by a simple inspection of TRACE images) and their contribution is more difficult to subtract.

The multi-wavelength observation allows for a cross-check of the results and for absolute calibration. Data in multiple TRACE EUV filter passbands allow for imaging and filter ratio diagnostics, SoHO/CDS data for spectroscopy, and Yohkoh/SXT for imaging in X-ray hotter passbands. The good visibility both in the $171 \AA$ and in the $195 \AA$ filter band ensures a good $\mathrm{S} / \mathrm{N}$ ratio for filter ratio (and therefore temperature) diagnostics. It is also visible in the $284 \AA$ filter band. Yohkoh/SXT data are of good quality, although mostly in a single filter band; a loop light curve can be derived from them. The $\mathrm{SoHO} / \mathrm{CDS}$ observation includes data in several spectral lines, with a good nominal coverage in the $4.5<\log (T)<6.5$ temperature sensitivity range.
It is important for our analysis that the data give information about the loop evolution. A long-lived and steady loop can be better studied, because it is close to physical equilibrium conditions. Although the selected loop eventually fades away, its observed intensity evolves slowly, and is nearly steady in a sequence of several TRACE images. We can then reasonably assume that the average properties of the loop plasma evolve slowly as well.

\subsection{The TRACE data analysis}

The campaign dataset includes four relevant TRACE data cubes. An overall number of $321024 \times 1024$ images for each filter are available, with exposure times between 1 and $39 \mathrm{~s}$, between 2 and $46 \mathrm{~s}$, and between 6 and $131 \mathrm{~s}$, for the $171 \AA$, the $195 \AA$ and the $284 \AA$ filter, respectively. In the 171 filter, the longest exposure time is the most frequent one ( 22 frames). In the $195 \AA$ filter, the exposure time is $28 \mathrm{~s}$ for 17 frames, $46 \mathrm{~s}$ for 6 frames. In the $284 \AA$ filter, 17 frames are taken with the longest exposure time.

The data have been processed using the standard IDL procedure trace_prep. A smaller region $(512 \times 512$ pixels $)$ of the whole field of view - the one enclosing the loop - has been extracted for analysis. For each filter band, the images have been coaligned with cross-correlation between the $512 \times 512$ images. We have removed the clearly corrupted frame 11 from all three datasets. In the $284 \AA$ Ailter frames 10 and 24 are clearly damaged by cosmic rays and removed.

\subsubsection{Background subtraction}

In order to apply standard diagnostic methods to derive physical quantities, such as the temperature, or even to apply more detailed loop models, we have to extract the emission from the loop and exclude any other contribution along the line of sight. Background subtraction may not be trivial in a region so rich in bright structures (e.g. Reale 2002; Schmelz et al. 2003). However, we have taken advantage of a particularly fortunate situation: the loop disappears at the end of the image sequence. The last images (around 10 UT) can then be used to derive a reliable background, in the assumption that the structures surrounding and crossing the selected loop along the line of sight do not change much during the observation sequence. The procedure has then been to simply subtract the last image from all the preceding images. This has been done for each filter image sequence.

This approach to subtract background has several advantages: it is in principle very accurate, if the loop under analysis is the structure which mostly varies in the observation; it is direct, with no use of interpolation; it is applied pixel by pixel, allowing us to derive "background-subtracted image" and therefore to have a visual feedback, and to analyze all loop pixels, instead of sampling at selected positions. As drawbacks, this method cannot take time-variations of the background emission into account and we cannot exclude that crossing structures vary during the observation. We have estimated the average time fluctuations during the observation by 

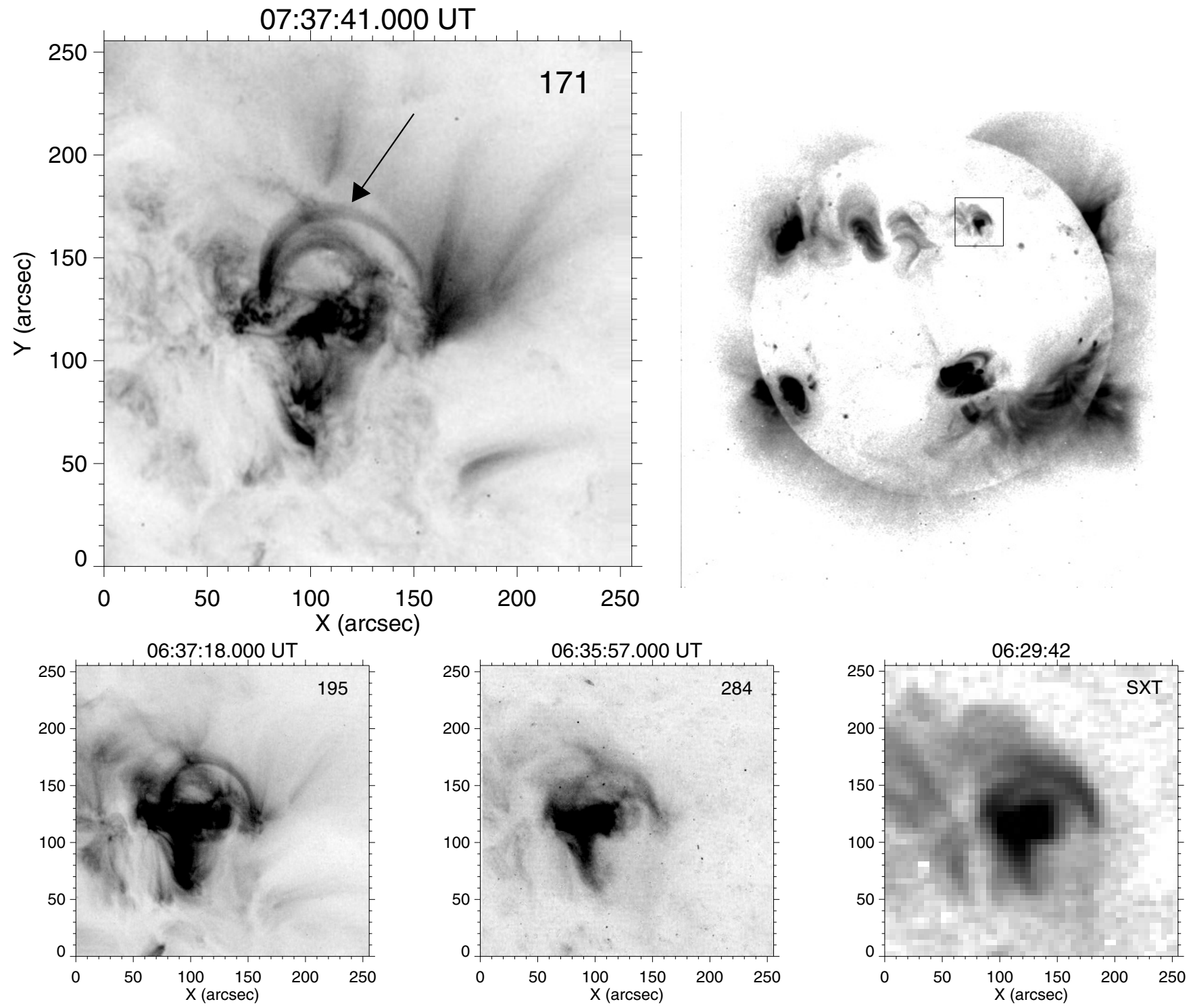

Fig. 2. The loop region $(256 \times 256$ arcsec $)$ in the passbands of the $171 \AA, 195 \AA$ and $284 \AA$ TRACE filters and in the Al/Mg/Mn sandwich filter of the Yohkoh/SXT. The grey scale is inverted and linear for all the TRACE images ( $\leq 8 \mathrm{DN} / \mathrm{s} / \mathrm{pix}, \leq 6 \mathrm{DN} / \mathrm{s} / \mathrm{pix}, \leq 2 \mathrm{DN} / \mathrm{s} / \mathrm{pix})$, and logarithmic for the Yohkoh image (between 10 and $150 \mathrm{DN} / \mathrm{s} / \mathrm{pix}$ ). In the $171 \AA$ image the loop is indicated with an arrow. The loop region is located in the inset of the Yohkoh/SXT full disk image (upper right).

computing pixel-by-pixel the standard deviation of the count rate. We obtain an average standard deviation of $13 \%$ both in the $171 \AA$ and in the $195 \AA$ filter band (not included in error bars), that we retain a reasonably acceptable value that validates our analysis. Although there is evidence of systematic effect due to co-evolving nearby structures, the overall validity of this approach is proven by the results obtained (Sect. 3).

\subsection{The Yohkoh/SXT data analysis}

The campaign data consist of two full-frame datasets, with nine relevant frames (full disk $512 \times 512$ pixels, each with a side of 4.9 arcsec). The exposure time of all frames is $2668 \mathrm{~ms}$. Only the first frame is taken in Al.1 filter band. All others are in the $\mathrm{Al} / \mathrm{Mg} / \mathrm{Mn}$ filter band. The frames are processed according to the standard Yohkoh procedure sxt_prep. Frames are co-aligned to each other and cross-aligned with the TRACE images (using the TRACE $512 \times 512284 \AA$ image at 6:57 UT) by means of the cross-correlation method. As for the background subtraction, the last frame has been subtracted from the previous ones in the $\mathrm{Al} / \mathrm{Mg} / \mathrm{Mn}$ filter band, analogously to what we have done with TRACE data. We will show results only for this passband.

\subsection{The SoHO/CDS data analysis}

CDS was observing from 07:13:15 to $12: 11: 58$ UT with 15 rasters in the region of the loop structure. Most of the rasters could not be included in the present analysis. The field of view of the first raster is not so large as to include the whole loop. The spectral ranges of the rasters from the third to the 13th are too narrow to scan the whole line 
Table 1. CDS file data.

\begin{tabular}{ccccccccc}
\hline \hline Filename & $t_{i}$ & $t_{f}$ & $X_{0}$ & $Y_{0}$ & $X$-width & $Y$-width & $X$-bins & $Y$-steps \\
& $(\mathrm{UT})$ & $(\mathrm{UT})$ & $\left({ }^{\prime \prime}\right)$ & $\left({ }^{\prime \prime}\right)$ & $\left({ }^{\prime \prime}\right)$ & $\left({ }^{\prime \prime}\right)$ & & \\
\hline s11107r00 & $07: 50: 09$ & $08: 46: 02$ & 208.0 & 489.3 & 243.8 & 243.8 & 60 & 72 \\
s11109r00 & $10: 23: 42$ & $12: 11: 58$ & 261.6 & 489.2 & 243.8 & 243.8 & 120 & 143 \\
\hline
\end{tabular}

Table 2. Observed spectral lines.

\begin{tabular}{lcc}
\hline \hline Line & $\begin{array}{c}\text { Wavelength } \\
{[\AA]}\end{array}$ & $\begin{array}{c}\log T \\
{[\mathrm{~K}]}\end{array}$ \\
\hline O V & 629 & 5.4 \\
Ca X & 558 & 5.9 \\
Mg IX & 368 & 6.0 \\
Mg X & 625 & 6.1 \\
Si X & 347 & 6.1 \\
Si X & 356 & 6.1 \\
Fe XII & 364 & 6.1 \\
Fe XIII & 348 & 6.2 \\
Si XII & 520 & 6.3 \\
Fe XIV & 334 & 6.3 \\
Fe XIV & 353 & 6.3 \\
Fe XVI & 360 & 6.4 \\
\hline
\end{tabular}

profiles. Thus, two rasters are useful to our analysis, those taken between 07:50:09 and 08:46:02 UT (s11107r00) and between 10:23:42 and 12:11:58 UT (s11109r00, Fig. 1). The latter has been used as background, since the loop had already faded out at that time. The filename, time, coordinates, spatial width and bin size of both rasters are listed in Table 1.

The standard CDS software was used to remove the spikes from the data and to calibrate them. Table 2 lists the lines shared by both relevant rasters and used for the present analysis.

The two rasters have different field of views, number of bins along $X$ and number of steps along $Y$ (see Table 1). TRACE and CDS images were co-aligned by cross-correlating the $512 \times 512$ TRACE $171 \AA$ image at 8:33 UT and the Mg IX $368 \AA$ image in the first CDS raster. We have co-aligned the images of the first raster to those of the second raster line per line. Following the same procedure as that used for the TRACE and Yohkoh data, the images of the second raster have been subtracted from the respective images of the first raster, for the lines shared by the rasters.

\section{Results}

\subsection{The loop region}

Figure 2 shows the loop region in the TRACE $171 \AA$, $195 \AA$, $284 \AA$ filter passbands and in the $\mathrm{Al} / \mathrm{Mg} / \mathrm{Mn}$ filter passband of Yohkoh/SXT, and its location on the solar disk. The loop is clearly visible in the TRACE 171 filter passband and is embedded in a region containing other bright structures. Some of them are well-defined, others not. There is a well-defined smaller concentric loop, which may itself deserve investigation. In the center of the field of view, there is a bright moss region, which has weaker filaments southwards. From the right footpoint of the selected loop, bright plumes mark the base of larger scale structures whose higher parts are invisible, probably because higher than the local pressure scale height. Another relatively bright structure appears to intersect the loop leftward of the apex. This structure may be really interacting with the loop, as illustrated below.

In the $195 \AA$ filter band, the loop region appears significantly different from the images in the $171 \AA$ filter band, and, in particular, the central moss region appears far brighter and extended, and the downward tail more prominent. However, even with no background subtraction, the loop is clearly visible in the $195 \AA$ filter band.

In the $284 \AA$ good images, the loop region appears more similar to that in the $195 \AA$ filter band than in the $171 \AA$ filter band, with a very prominent central moss region, but generally more diffuse, as typically occurs in the $284 \AA$ filter band. The sensitivity in this filter is more than one order of magnitude lower than in the $171 \AA$ filter band (Handy et al. 1999) and would require an equivalent increase of exposure time to obtain equivalently contrasted images. Instead, the exposure time is increased by a factor 3 to 5 ; the lower $\mathrm{S} / \mathrm{N}$ ratio may explain, at least in part, the lower contrast and definition of the structures in the region. In the initial images, a loop structure similar to those visible in the other two filter bands is visible, although more diffuse and weaker. The central moss region is even more dominant in the $284 \AA$ images.

In the Yohkoh/SXT Al/Mg/Mn filter passband, the loop region appears similar to the region in the TRACE $284 \AA$ filter band. In particular, we note the bright feature at the center of the field of view, and the loop arcade above it.

\subsection{The loop evolution}

The loop evolution during the campaign is summarized in the sequence of four background-subtracted images shown in Fig. 3, in the TRACE $171 \AA, 195 \AA, 284 \AA$ filter passbands and in the Yohkoh/SXT Al/Mg/Mn filter passband, at corresponding times. In these images, all negative values have been set to zero to emphasize the brightness excesses over the last image. The images show that also other structures are variable in the loop region, e.g. the central moss region, the inner loop and other outer loop structures. The loop is visible with better contrast after background subtraction, in all TRACE and SXT passbands, in the initial phases of the observation, and in several CDS lines (see Fig. 9). Since it is well inside the solar 

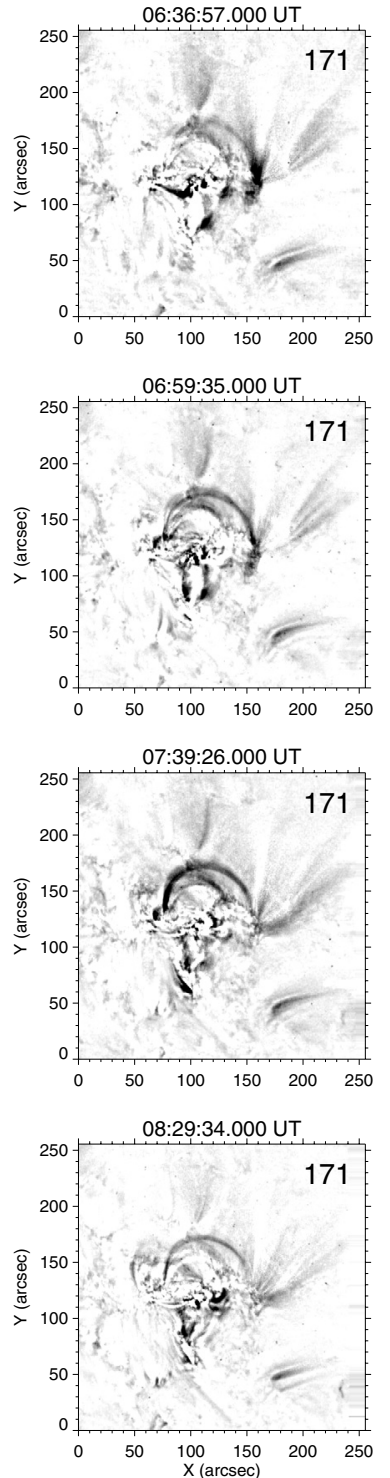
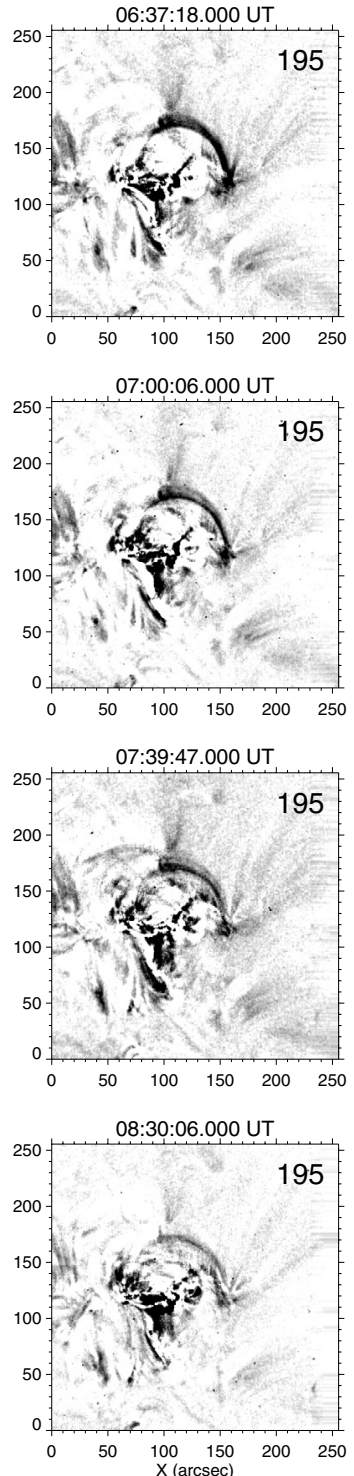
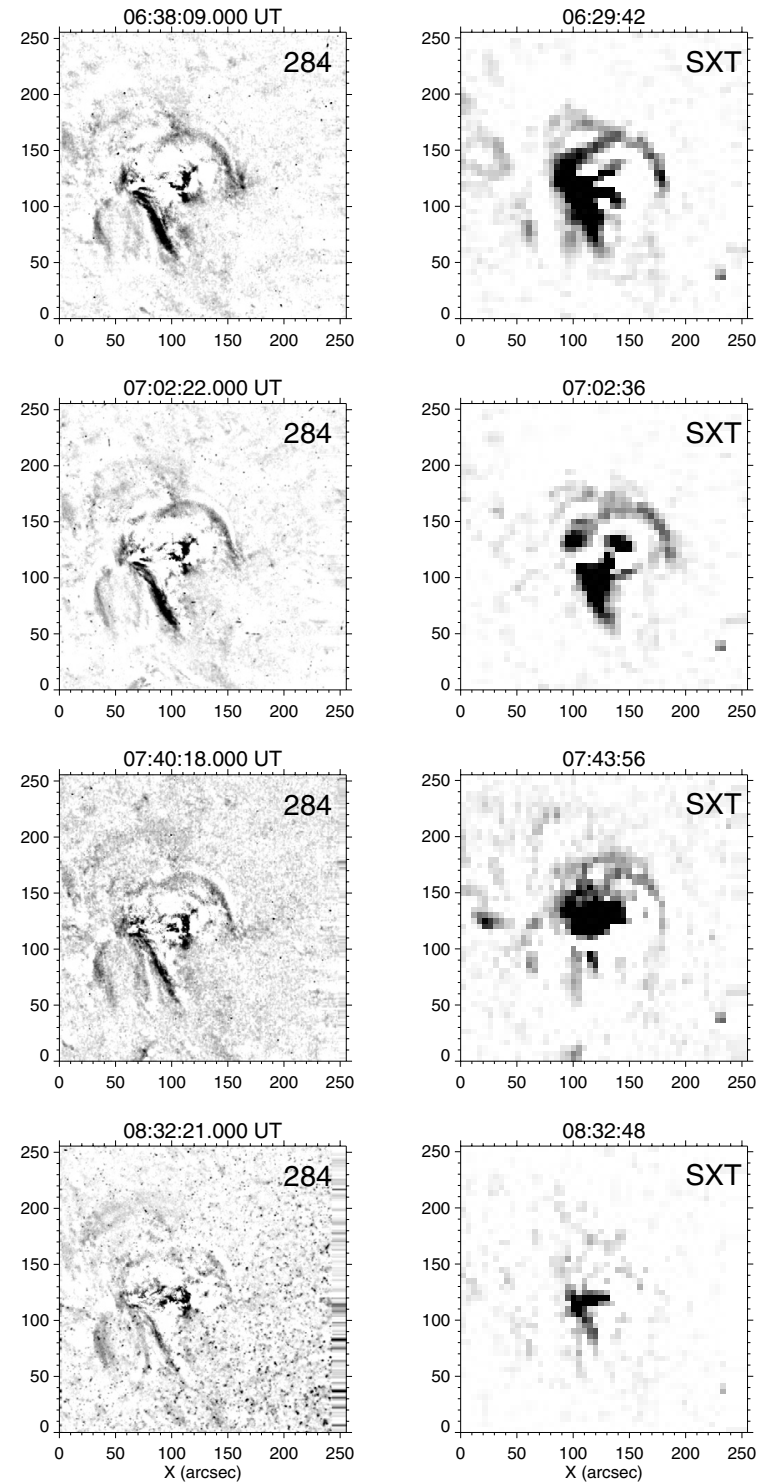

Fig. 3. Background-subtracted image sequence of the loop region $(256 \times 256$ arcsec) in the passbands of the $171 \AA$, $195 \AA$ and $284 \AA$ TRACE filters and in the $\mathrm{Al} / \mathrm{Mg} / \mathrm{Mn}$ sandwich filter of the Yohkoh/SXT, from 6:31 UT to 8:31 UT. The grey scale is inverted and linear for all the images ( $\leq 8 \mathrm{DN} / \mathrm{s} /$ pix, $\leq 6 \mathrm{DN} / \mathrm{s} /$ pix,$\leq 2 \mathrm{DN} / \mathrm{s} /$ pix,$\leq 150 \mathrm{DN} / \mathrm{s} /$ pix). Negative values have been set to zero.

disk and its shape well approximates a semi-circle, it is probably inclined on the surface.

In the TRACE $171 \AA$ filter passband, the selected loop is visible from the beginning, but it becomes completely visible half an hour later, at about 7:00 UT, and bright at maximum at 7:30 UT. It begins to fade significantly about one hour later and is no longer visible at about 9:15 UT. In the $171 \AA$ images, the right leg of the loop is initially the brighter. Later on, the brightness becomes more uniform along the loop and eventually the left leg becomes the brighter, before the whole structure fades away.

In the TRACE $195 \AA$ filter passband, the evolution is slightly different, in that the loop appears to be monotonically and uniformly fading out toward the end of the observation. The loop appears to be truncated quite abruptly on the left side for about $1 / 4$ of its length, and to be bright again close to the left footpoint. We have checked that in the dark region the background is particularly intense due to a bright intersecting structure still present in the last image. The presence of this bright structure may affect the background subtraction there. The right side is initially quite uniformly bright, with a "halo" on the outer shell. We have checked that the $171 \AA$ loop overlaps the brightest arch of the $195 \AA$ background-subtracted images (see also Sects. 3.3 and 3.4).

In the TRACE $284 \AA$ filter passband, the loop structure is quite faint and overlaps only the inner part of the loop of the other two passbands. It fades out rapidly. The right leg is quite uniformly bright, the left leg has a gap close to the footpoints.

In the Yohkoh/SXT Al/Mg/Mn filter passband, a loop structure appears in the initial background-subtracted images at a similar location and with a similar shape as the loop visible in the TRACE passbands, in spite of the different passband. The right leg of the Yohkoh/SXT loop overlaps well with the right leg of the TRACE loop. As time progresses, the loop becomes 
fainter and fainter, and it is barely visible at 7:44 UT and no longer at 8:30 UT. The bright region southward of the loop also appears to be variable and fading to the end of the observation.

From an inspection of the TRACE images of the loop region before the beginning of the campaign, we have checked that the loop is present and bright from about 2 UT (see also Sect. 3.3.2).

\subsection{Loop data analysis}

Our next step is to analyze the emission inside the loop and the related diagnostics. We define a strip enclosing the loop in the TRACE $171 \AA$ filter passband, down to the visible footpoints and even beyond them, and divide it into sectors, as shown in Fig. 4. We have analyzed strips of different widths; here we show results for a width of 10 pixels, which encloses the bulk of the loop and represents a good compromise: a thinner strip encloses too few pixels for good enough statistics and may be severely affected by slight alignment errors; a wider strip may not warrant enough coherence across the loop, i.e. it may include loop strands in too different conditions to define average properties. With the choice of a length of 10 pixels, we end up with 27, almost square, sectors. Starting from the left extreme the first three sectors are beyond the visible loop, and the fourth one partially includes the loop extreme. The fifth sector can be reasonably considered as the first visible piece of the loop. On the other side, we may include all but the last sector.

The DN counts are extracted from each sector, at corresponding locations in the different passbands of TRACE and Yohkoh/SXT, after cross-aligning the images, independently of the degree of overlap of the loop structure in the different passbands (i.e., the bins have the same solar coordinates in the different passbands to within the coalignment uncertainties). The SXT images have been rebinned to the same pixel size of TRACE, to use the same TRACE strip and sectors for the emission extraction.

\subsubsection{Profiles along the loop}

Figure 5 shows the background-subtracted emission profiles along the loop in the three TRACE filter passbands and in the Yohkoh/SXT $\mathrm{Al} / \mathrm{Mg} / \mathrm{Mn}$ filter passband at the same times as in Fig. 3. The error bars are computed as the sum in quadrature of the error on the total photon counts and of the error on the background photon counts. The dashed vertical lines mark the approximate boundaries of the bright structure which intersects the loop (see Sect. 3.1). This structure produces a clear bump in most of the emission profiles along the loop (and in all passbands); since it appears to evolve together with the loop, it probably interacts with the loop. However, it should not be part of the loop and we will exclude it from the loop analysis. We see in Fig. 5 that the global emission level is much lower than that of the unsubtracted profiles in all passbands - of the order of a half or even less - indicating that the background emission is indeed a significant fraction of the total emission from the region.
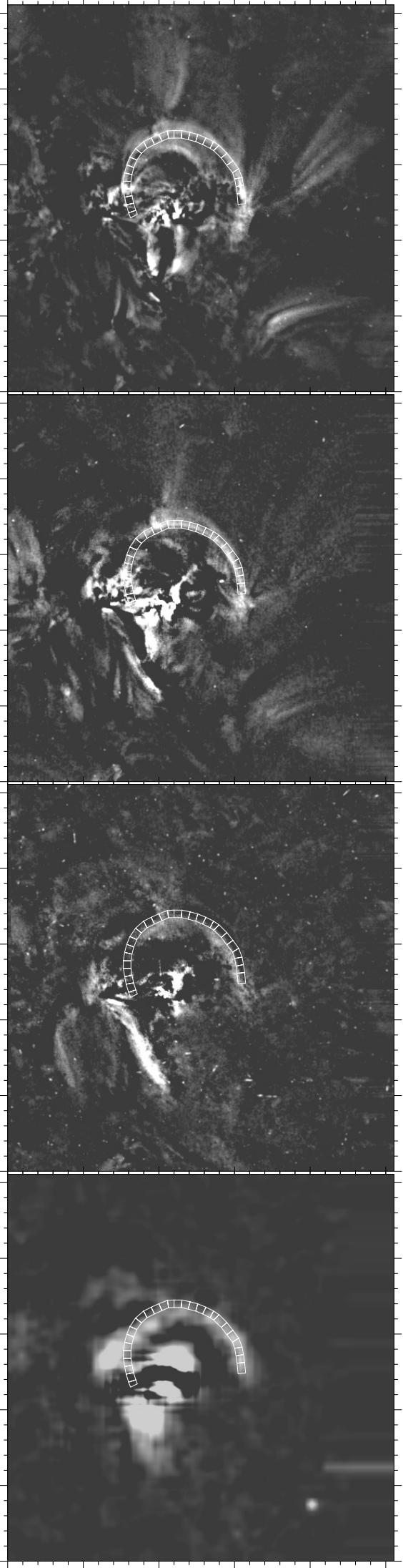

Fig. 4. Counts for loop analysis are extracted from the (white) strip on the images (second row in Fig. 3 shown with an inverse gray scale), 10 pixels wide. The count profiles are built after dividing the strip into sectors (10 pixels long). 


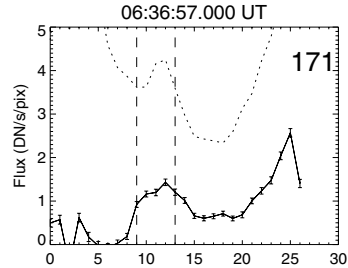

06:59:35.000 UT

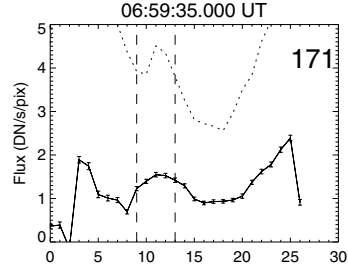

07:39:26.000 UT

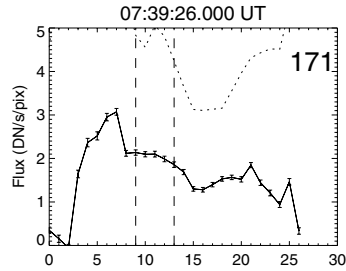

08:29:34.000 UT

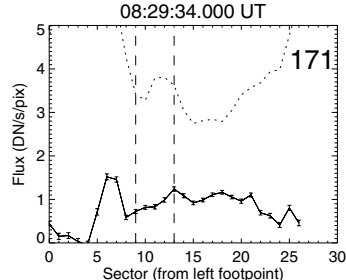

06:37:18.000 UT

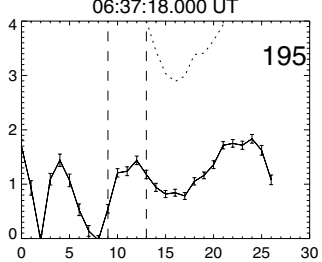

07:00:06.000 UT

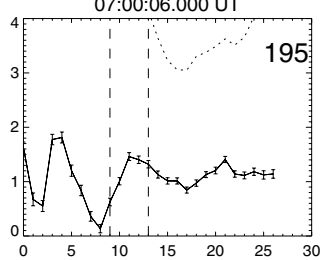

07:39:47.000 UT

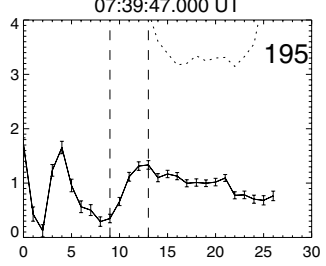

08:30:06.000 UT

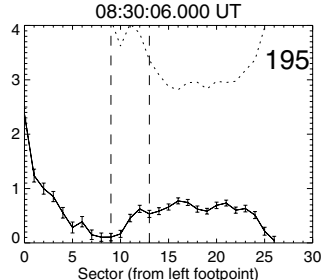

06:38:09.000 UT

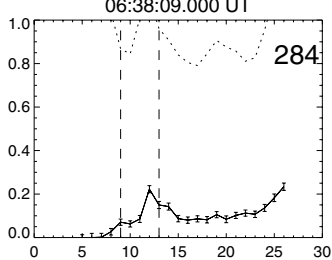

07:02:22.000 UT

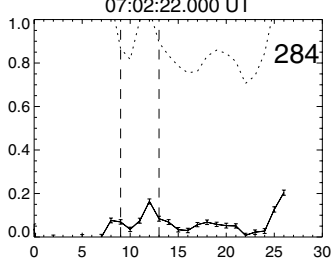

07:40:18.000 UT

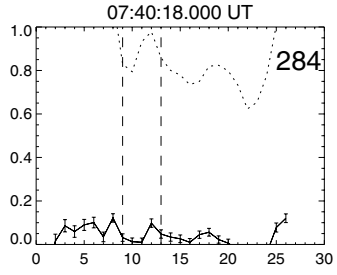

08:32:21.000 UT

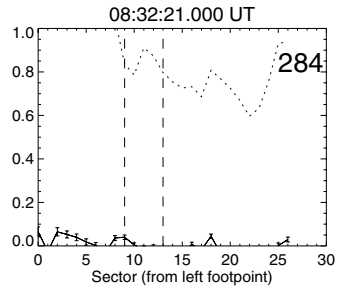

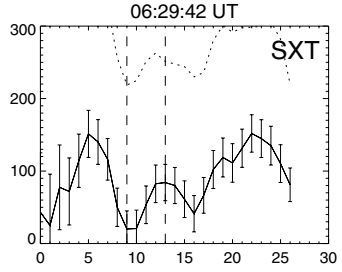

07:02:36 UT

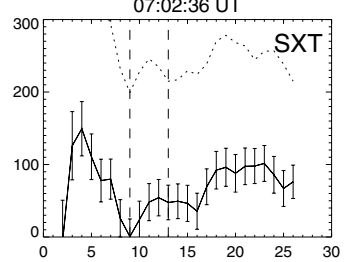

07:43:56 UT

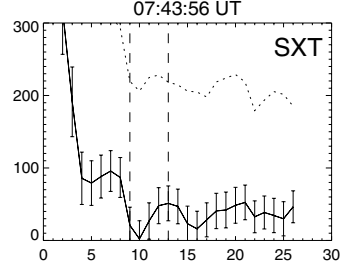

08:32:48 UT

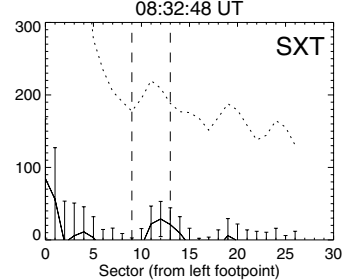

Fig. 5. Background-subtracted emission profiles (solid, data points) along the loop in the $171 \AA$, $195 \AA$ and $284 \AA$ TRACE filter passbands and in the Yohkoh/SXT Al/Mg/Mn filter. The unsubtracted profiles are also shown (dotted lines). The dashed vertical lines bound the region in which a bright irregular structure intersects the loop along the line of sight.

At the beginning, the loop emission comes mostly from its right leg in the $171 \AA$ filter passband, and is more uniform in the $195 \AA$ and $284 \AA$ filter passbands. In the Yohkoh/SXT initial profile the emission is significant in the loop legs and low around the apex. The emission profiles confirm that, in the $171 \AA$ filter passband, the emission evolution is asymmetric: the left leg of the loop progressively brightens and the right leg correspondingly fades. At 7:00 UT the emission profile is almost flat along the loop. At 7:40 UT the left extreme is bright and the other is dark. Then also the left leg becomes fainter, and after 8:30 UT the whole loop is virtually dark, although there is low and decreasing residual emission. The bump becomes less and less prominent with time and in the second and third part of the observation it becomes just a slight change of slope in the emission profiles.

The background-subtracted $195 \AA$ Afilter profiles show a dip on the left side (between sectors 5 and 10). This feature is also present in the background-subtracted images (Fig. 3) and is probably due to a co-evolving intersecting structure (Sect. 3.2). The left loop footpoint and the whole right leg are bright. In this filter the loop emission decreases with time as a whole. The $284 \AA$ filter profiles are very low. The left leg is never as defined and bright as in the other two filter bands. The right leg appears to be brighter in the first two profiles. Globally, the profiles decay with time; the emission becomes negligible after 7:00 UT. Also in the Yohkoh/SXT passband, the loop emission decreases with time. The regions close to the footpoints appear to be both brighter than the central loop region and the emission looks overall quite symmetric in the first two profiles. Thereafter it may be compatible with zero emission as in the TRACE 284 Å filter passband.

\subsubsection{Light curves}

We can build the light curve in the various filter passbands by integrating the emission along the strips. The emission is integrated from sector 4 to sector 26. For comparison we compute also the evolution of the emission integrated only on the right half of the loop (sector 14 to 26). We will not show and comment on the unsubtracted light curves. The backgroundsubtracted light curves both in the whole and in half of the loop are shown in Fig. 6 (average DN rate per pixel).

In the TRACE $171 \AA$ filter band, the light curve of the whole loop first increases, with a peak about one hour after the beginning of the observation, and then monotonically decreases to zero level at $t \sim 12 \mathrm{ks}$. The e-folding decay time of the whole loop is $\tau_{171}=2.5 \pm 0.4 \mathrm{ks}$ since the maximum. Note that the free cooling time (Serio et al. 1991) expected for 

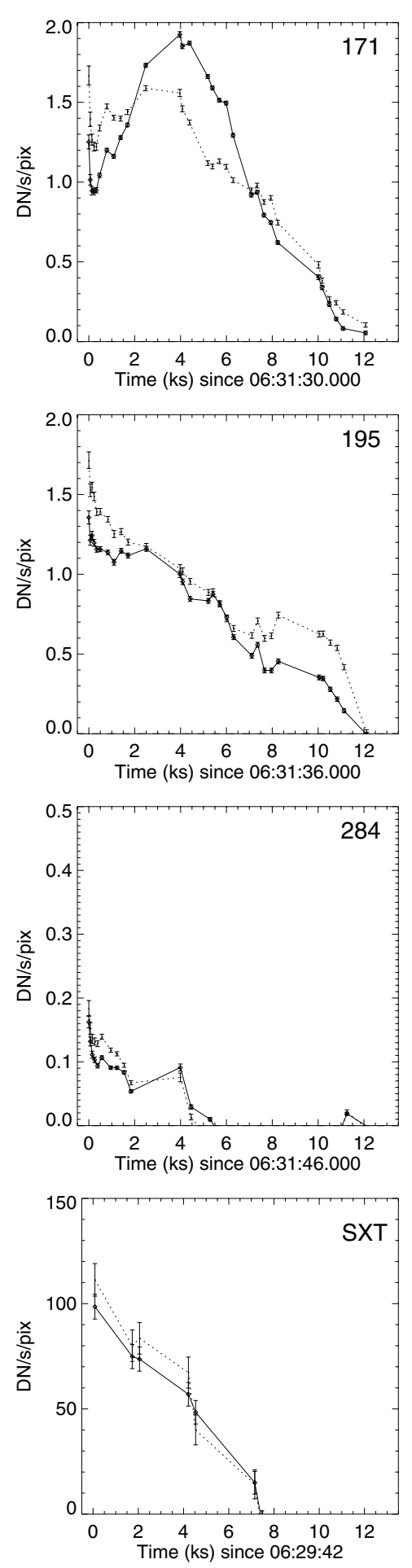

Fig. 6. Background-subtracted light curves integrated along the whole loop strip (solid line) and on the right loop half only (dashed line) in the TRACE $171 \AA, 195 \AA$ and $284 \AA$ filter passbands, and in the Yohkoh/SXT Al/Mg/Mn filter passband.

a loop of half-length $L \approx 4.2 \times 10^{9} \mathrm{~cm}$ at $1 \mathrm{MK}$ is about $1.6 \mathrm{ks}$, i.e. a shorter timescale. The cooling times will be even shorter than this if the loops are either over-dense or under-dense, as is typically the case for TRACE and Yohkoh loops, respectively (e.g., Winebarger et al. 2003a). The emission from the right leg appears more constant in the first part of the observation, and then decreases with the total loop.

The light curve decays monotonically in all the other passbands, with very similar trends in the whole and in the right half of the loop. The e-folding times are $\tau_{195}=6.1 \pm 0.7 \mathrm{ks}$, $\tau_{284}=2.9 \pm 0.7 \mathrm{ks}, \tau_{\mathrm{SXT}}=4.0 \pm 0.7 \mathrm{ks}$. The slowest decay is in the $195 \AA$ band, which is probably the one that detects most completely the loop plasma cooling. In this band there are slight increases of the emission of the whole and of the right half of the loop at times $t \sim 2 \mathrm{ks}$ and $t \sim 8 \mathrm{ks}$, respectively. In the TRACE $284 \AA$ band and in the Yohkoh/SXT band, the decay is quite fast and the emission becomes negligible after about $4 \mathrm{ks}$ and $7 \mathrm{ks}$, respectively.

We have checked that the loop emission is relatively steady, with fluctuations, in the four hours preceding the campaign, with an average emission of $0.8 \mathrm{DN} \mathrm{s}^{-1} \mathrm{pix}^{-1}$ in both the $171 \AA$ and $195 \AA$ filter bands, with a standard deviation of 0.2 and $0.1 \mathrm{DN} \mathrm{s}^{-1} \mathrm{pix}^{-1}$, respectively. The beginning of the campaign approximately corresponds to the time of maximum loop emission in the $195 \AA$ band. The loop emission in the SXT image at 5:26 UT is significantly lower than the one at 6:29 UT (shown in Fig. 3) approximately by the same amount ( 50\%) as the corresponding emission in the TRACE $195 \AA$ filter band.

\subsection{The TRACE filter ratios}

The temperature diagnostics from the ratio of the count rates in thin passband filters such as those of TRACE suffer from possible uncertainties, because of possible unpredictable variations of the element abundances of the few lines emitted in the bands, of the temperature stratification along the line of sight, and of the non-monotonic dependence of the filter ratio on the temperature.

The problem is made even more difficult by the superposition of different incoherent structures along the line sight. If this "background" is not subtracted properly, an artificial emission offset is created, which will flatten the filter ratio profile along the structure, i.e. it will tend to make the ratio closer to unity everywhere ${ }^{1}$. Therefore, one has to compute the filter ratio after background subtraction. Although the loop data in the TRACE $284 \AA$ filter passband have some statistical significance in the initial part of the observation, we have found the $284 / 195$ filter ratio to yield large uncertainties and we prefer not to comment on it. We have no way to obtain backgroundsubtracted images in two Yohkoh/SXT filter passbands (only in the $\mathrm{Al} / \mathrm{Mg} / \mathrm{Mn}$ one) and we will address no filter ratio diagnostics for Yohkoh data. We will therefore concentrate on the TRACE 195/171 filter ratio.

As a first step, we obtain filter ratio maps, by computing the pixel-to-pixel ratio of whole images, taken almost simultaneously (few seconds difference) shown in Fig. 7 at the same times as in Fig. 3. The filter ratio is computed only where the pixel count rate is above 0.5 and $0.2 \mathrm{DN} / \mathrm{s}$ in the $171 \AA$ and $195 \AA$ filter bands, respectively, i.e. where there is enough $\mathrm{S} / \mathrm{N}$ ratio.

\footnotetext{
${ }^{1}$ Unless high contributions from cold plasma are present.
} 

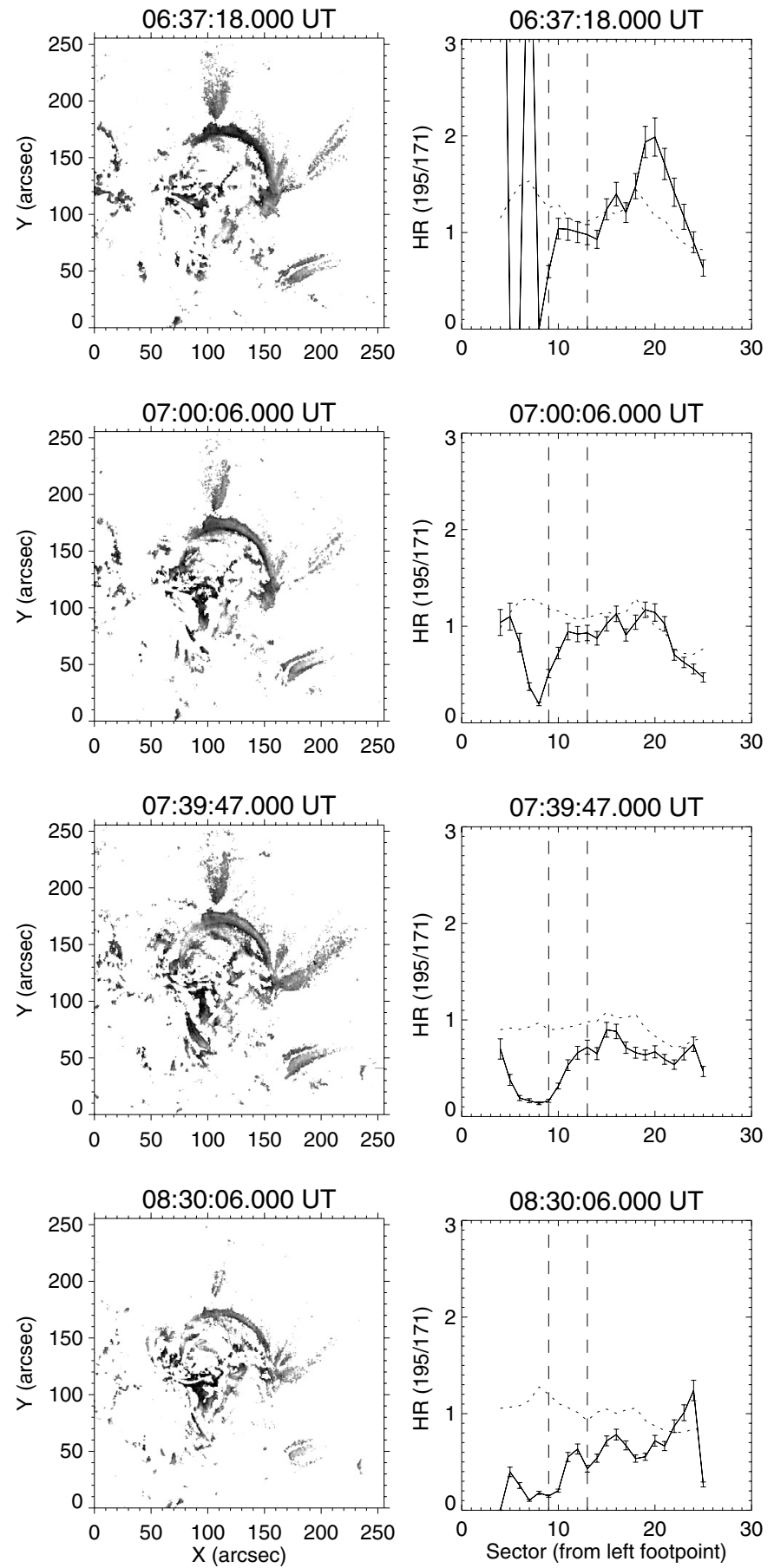

Fig. 7. Left: background-subtracted 195/171 filter ratio maps of the loop region $(256 \times 256 \mathrm{arcsec})$ from $6: 30$ UT to 8:30 UT. The grey scale is logarithmic between 0.1 (white) and 2 (black). The ratio is computed only where the pixel count rate is above 0.5 and $0.2 \mathrm{DN} / \mathrm{s}$ in the $171 \AA$ and $195 \AA$ A filter bands, respectively. Right: backgroundsubtracted filter ratio $195 / 171$ profiles along the loop. Unsubtracted profiles are also shown (dotted lines).

Also thanks to the screening devised above, the loop shows up clearly in the filter ratio maps, especially at early times, when the $195 \AA$ signal is high. This is an indication that the loop imaged by the $171 \AA$ filter is well aligned to the loop imaged by the $195 \AA$ filter, supporting that both filters are detecting the same plasma. The filter ratio distribution appears full along the right leg, a gap (a zone of low value) is present in the left leg. In the chosen grey scale, darker tones indicate higher filter ratio and, probably, hotter plasma. In the initial frames, where the signal is higher, the filter ratio appears to be structured, both across and along the loop. In the first map, higher (darker) values are present in the inner shell and in the region of the loop apex. In the second and third map, both the outer and inner shells of the loop appear to be darker. The loop color clearly shifts to paler tones as time passes, indicating that the structure is cooling while fading. The right footpoint appears to be at lower filter ratio values.

The right strip of Fig. 7 shows the filter ratio profiles at the same times as the filter ratio maps (left strip). All the profiles are included along most of the loop in the range between 0.2 and 5, i.e. $6 \leq \log T \leq 6.2$ (according to the standard TRACE software). The first filter ratio profile has a peak around sector 20, i.e. in the middle of the right half of the loop. This feature persists for about $5 \mathrm{~min}$ and may be a local temperature maximum. On the left loop side, there are low filter ratio values, forming a well-defined dip. This dip is probably sharper than it should be, because its right side is made steeper by the presence of the bright intersecting structure. Given the expected filter ratio to temperature relationship at temperatures below 1 MK (e.g. Lenz et al. 1999; Testa et al. 2002), the dip may be the signature of a monotonic decrease of the temperature from the loop top toward the left footpoint. However, this indication is to be taken with care, since the filter ratio dip is mostly caused by a dip in the $195 \AA$ profile (Fig. 5), which may be an artifact of the subtraction of a bright feature in that region, as shown in Sect. 3.2. At intermediate times the filter ratio peak in the right loop side disappears and the profiles are flatter there. At 8:30 UT, another clear peak appears again on the right leg, closer to the footpoint. Overall, the average emission level clearly progressively decreases.

For comparison, Fig. 7 also shows the profiles obtained with no background subtraction. All unsubtracted profiles are flat and equal, all around a value of filter ratio of one, and show no evolution.

Figure 8 shows the evolution of the background-subtracted filter ratio averaged over the loop (between sectors 8 and 25) and around the loop apex (between sectors 13 and 20). The latter is systematically higher, due to the non-uniform distribution of the filter ratio along the loop. Both ratios clearly decrease with time: this is evidence that the loop is gradually cooling on average.

\subsection{The CDS data}

Figure 9 shows the images in five spectral lines detected with CDS during the first raster and the same images after the background subtraction. The rastering roughly takes the time between the last two panel rows in Fig. 3. In the difference images, the loop shows up in a few lines. It is best visible in the Mg IX $368 \AA$ line. Most of it is visible also in the Mg X $625 \AA$ line and in the Si X lines (especially the $347 \AA$ one). The left leg is bright in the Ca X $558 \AA$ line. The loop is barely visible in the Fe XII $364 \AA$ line, and not visible at all in the remaining 


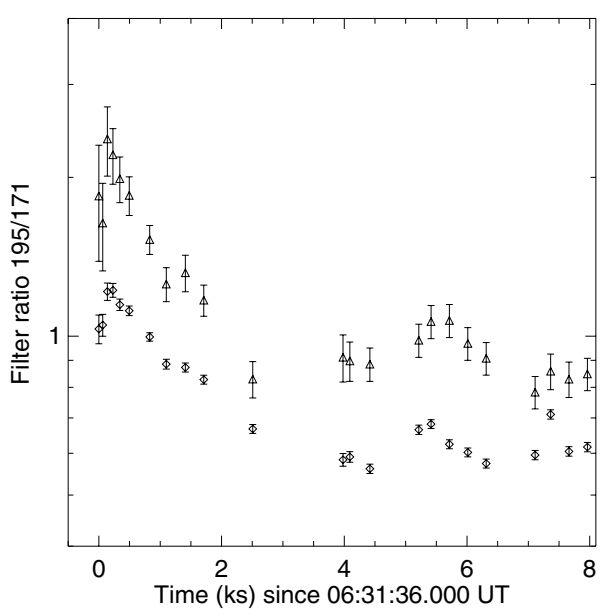

Fig. 8. Time evolution of the filter ratio $195 / 171$ averaged over the loop (between sectors 8 and 25, diamonds) and the maximum ratio value taken between sectors 13 and 20, i.e. the right leg of the loop (triangles).

lines. The hottest lines of Fe XIV and Fe XVI may be detecting the right end. In summary, we see the loop in the lines with peak formation temperature in the range $5.9 \leq \log T \leq 6.1$, at best for $\log T=6.0$, and we do not see it in the lines outside this range. We also see the left leg as the brighter one. All this is consistent with the loop as it appears in the $171 \AA$ Af filter band in the same time period (around 8 UT, see also Fig. 13).

Figure 10 shows a set of four images for the $\mathrm{Mg}$ X $625 \AA$ line: the images of the first and second raster (the background image) and the difference images, the second one including the loop contour as obtained from the TRACE data. The coarser spatial resolution of CDS images makes the loop less defined than in the TRACE images.

For the analysis of the emission from the loop plasma, in order to match easily the TRACE data and to use the same loop outline, the CDS images have been rebinned to the pixels size of TRACE. The loop has been divided into 13 sectors in the rebinned difference images, and the average line intensities have been computed in each sector. The loop sectors in CDS images are different from those chosen to analyze the loop in the TRACE images - approximately twice as long (Sect. 3.3). Few CDS sector pixels yield negative counts; they have been set to zero. Figure 11 shows the intensities in five lines along the loop. The error bars have been computed as the standard deviation of the photon counts within each CDS sector. The profiles show a well-defined trend in the lines where the loop is visible in the difference images, i.e. from Ca X 558 Å to Fe XII $364 \AA$ A: there is an emission peak around sectors 1 to 3 and then the emission decreases more or less gradually toward the right leg of the loop. In the cool O V $629 \AA$ line, the only significant feature is a bump at the left extreme. There is no clear link between this bump and the loop emission. The profile in the Mg IX $368 \AA$ line overall appears similar to the background-subtracted profile in the TRACE $171 \mathrm{~A}$ filter band at 7:39 UT (Fig. 5, see also Fig. 13). In the hottest lines (Si XII, Fe XIV and Fe XVI) the profiles show weak features on the right side of the loop region, which may be evidence of some hot plasma there. In the
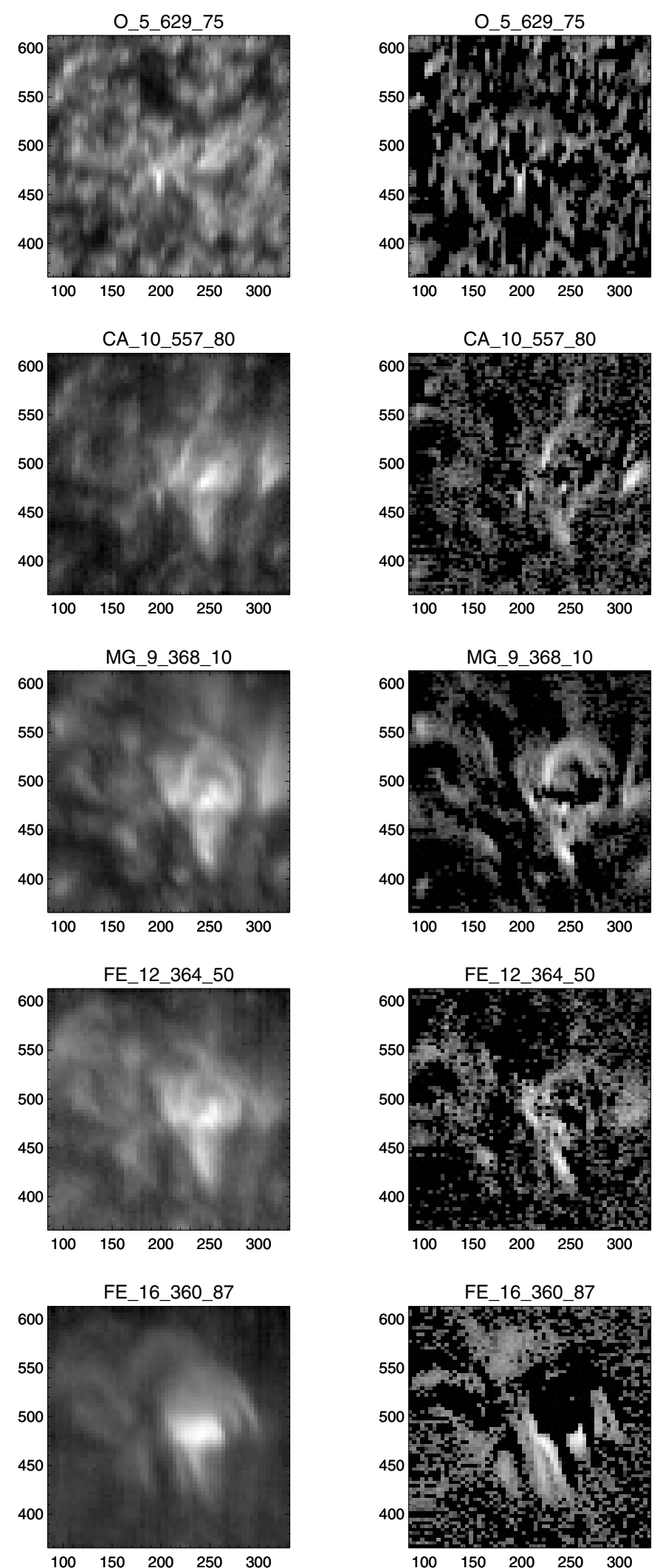

Fig. 9. SoHO/CDS images of the loop region in five spectral lines during the raster between 07:50:09 UT and 08:46:02 UT before (left column) and after (right column) the background subtraction. The image coordinates are in arcsec. 

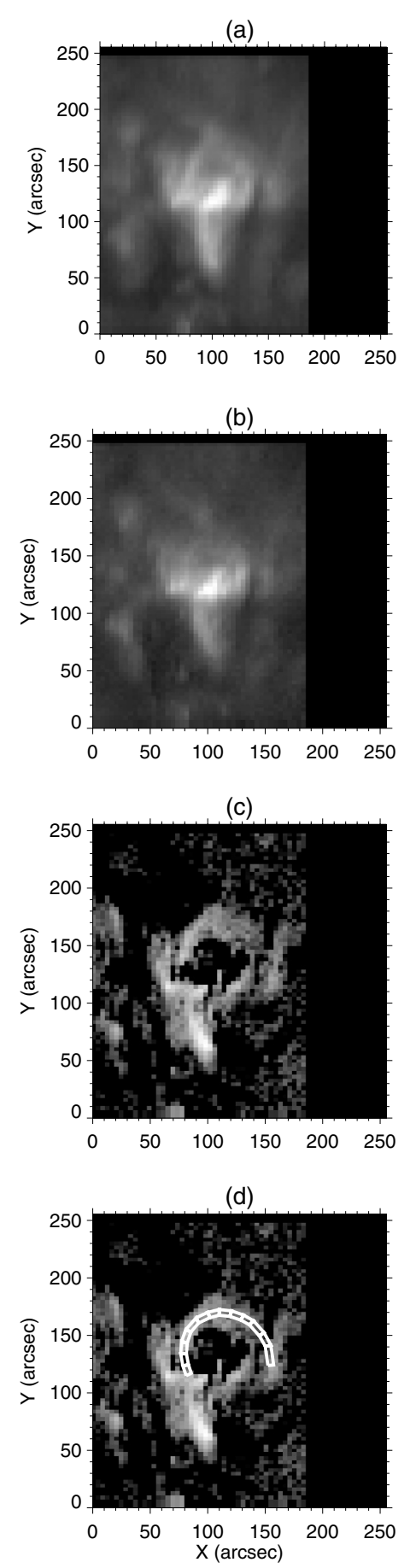

Fig. 10. The loop as seen by CDS in the Mg X $625 \AA$ line. The the image of the loop is in panel a), the background image in panel is in panel b). Panel c) shows the background subtracted image and panel d) the same image with the outline of the loop as obtained from TRACE images.

lines peaking around $1 \mathrm{MK}$, the profiles are consistent with a more luminous left leg. This appears to be consistent with the emission distribution along the loop observed on average in the $171 \AA$ filter band of TRACE in the corresponding time interval (lower two plots in Fig. 5).
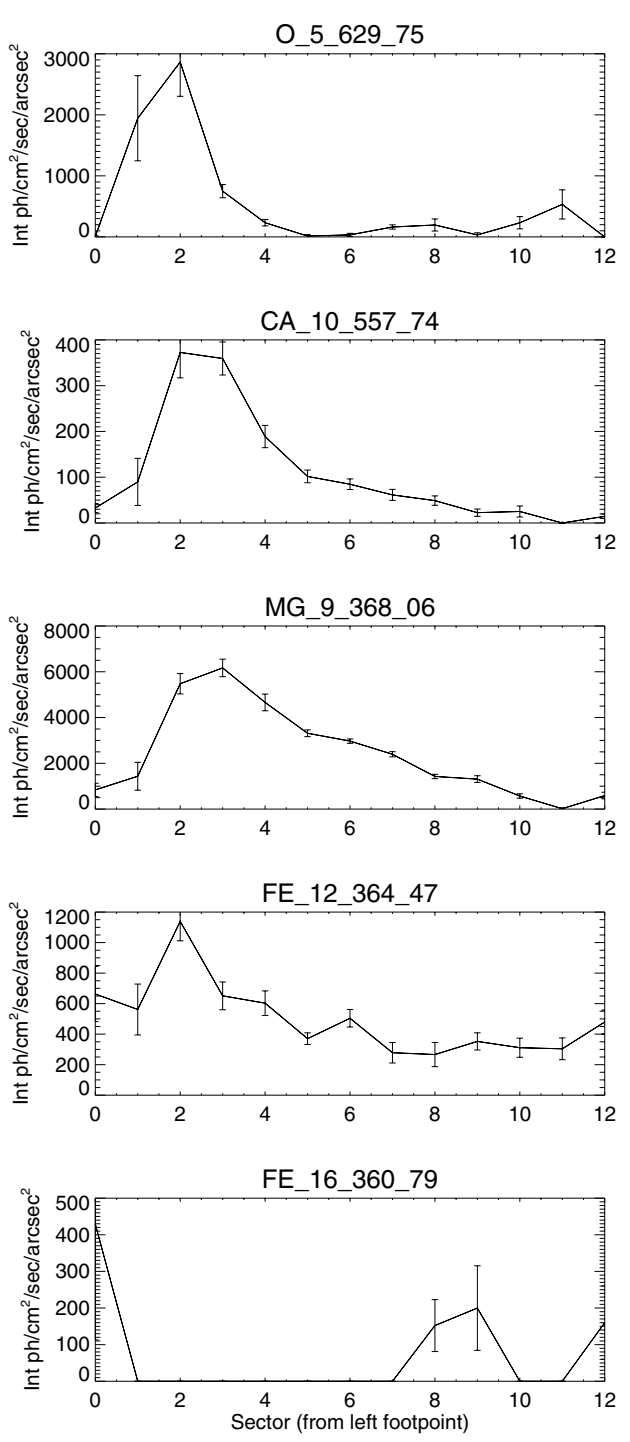

Fig. 11. Line intensities as function of the position (sector) along the loop. The loop has been divided in thirteen sectors as shown in Fig. 10.

\subsubsection{Emission measure}

Line emissivities were computed using CHIANTI V4.0.2 with ionization equilibrium fractions from Mazzotta et al. (1998) and photospheric abundances. Figure 12 shows the emission measure loci diagrams of five CDS sectors along the loop, namely CDS sectors 2, 4, 7, 9 and 10 (see Fig. 11 for some of the line intensities), roughly corresponding to TRACE sectors 4, 8, 14, 18 and 20 (Sect. 3.3). CDS sectors 2 and 4 are in the left leg, CDS sector 7 around the loop apex, and CDS sectors 9 and 10 in the right leg. The curves are shown only for those lines in which the emission in the CDS sector is larger than zero. Among the lines we used in Fig. 12, Li-like (Mg X, Si XII) and Na-like ( $\mathrm{Ca} \mathrm{X}$ ) lines are those with the largest uncertainties. As shown by Landi et al. (2002a,b), the emission measures of Li-like lines are lower by a factor of 2 when compared to the values obtained with other spectral lines. For the Na-like emissivities the status is less clear. Na-like lines emissivities have been found to be higher by a factor of 2 

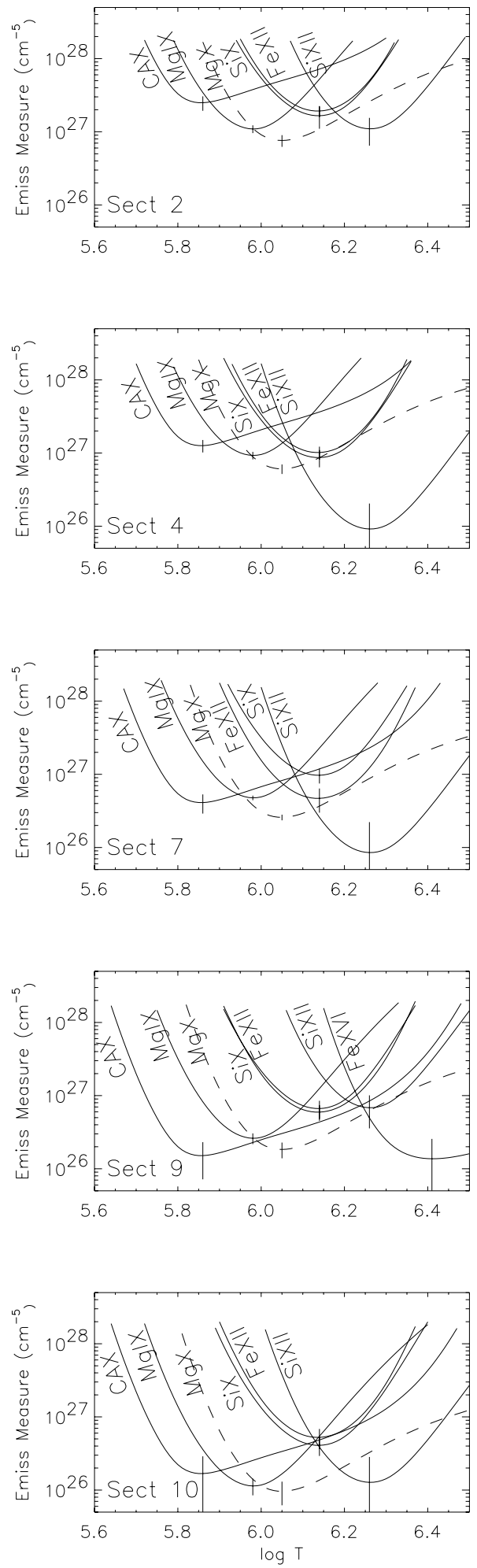

Fig. 12. Emission measure distribution in five CDS sectors $(2,4,7$, 9,10 ) of the loop. The Mg X $625 \AA$ line (dashed line) appears to be inconsistent with the others.

(Landi et al. 2000a) when compared with SUMER spectra. A good agreement with the other ions has been found in comparison with CDS spectra (Landi et al. 2002b). We have found that the Mg X $625 \AA$ line is clearly inconsistent with all others in all sectors (dashed line in Fig. 12). Similar problems with the
Mg X $625 \AA$ line have been pointed out in a specific spectral study made on solar data using the ADAS atomic and spectral model (Lanzafame et al. 2005). We therefore decided to discuss the emission measure distributions without considering this line.

As a result, in Fig. 12 CDS sector 9 appears to be the hottest, with a peak around $\log T \approx 6.25$ and with a relatively broad distribution on the cool side down to $\log T \approx 6.0$. CDS sector 10 is somewhat cooler, well peaked around $\log T \approx$ 6.15, and CDS sectors 4 and 7 are even cooler with peaks at $\log T \approx 6.05$, and again with a relatively broad distribution towards the cool side. The situation of CDS sector 2 is less clear. This CDS sector is located very close to the loop left footpoint and appears bright in the Si XII line probably because of other bright overlapping structures. In the light of this, the panel for CDS sector 2 may be compatible with a relatively cool distribution at $\log T \approx 6.1$, not different from CDS sectors 4 and 7 , and the $\mathrm{Ca} X$ line may suggest the presence of significant cooler contributions. This may be consistent with the bright left leg of the loop in the TRACE $171 \AA$ images (Fig. 7) and in the cool lines CDS images (Fig. 9). Overall, the figure suggests a trend of increasing temperature from the footpoints to CDS sector 9, located in the right leg of the loop.

Knowing that the raster took about $1 \mathrm{~h}$ to span an $\mathrm{X}$ distance of $240^{\prime \prime}$, we obtain that the time difference to raster from sector 4 to $10\left(50^{\prime \prime}\right)$ is about $12 \mathrm{~min}(0.7 \mathrm{ks})$. This is certainly too small a time lapse to determine significant variations of this loop, and we conclude that we are seeing the spatial structure of the loop.

The emission measure appears to be the highest in CDS sector $2\left(\sim 2 \times 10^{27} \mathrm{~cm}^{-5}\right)$ and to decrease progressively toward the right end of the loop $\left(\sim 5 \times 10^{26} \mathrm{~cm}^{-5}\right)$.

\section{Discussion}

\subsection{Data analysis and background subtraction}

In this work we present the analysis of a coronal loop imaged in multiple filters and spectral lines over part of its lifetime. The loop was selected on TRACE images. Our analysis collects the information coming from the time-evolution of the loop region in three TRACE passbands, and in one Yohkoh/SXT passband at overlapping times, and two SoHO/CDS rasters in twelve relevant lines, one during the TRACE observation, the other soon after. The selected loop is bright, lies on the disk and part of it is well isolated from other bright structures. The fact that we image a complete loop structure and monitor part of its evolution provide further constraints of coherence for the data interpretation.

Since the loop fades out at the end of the TRACE and Yohkoh observations and is absent in the second CDS raster, we could use the final TRACE and Yohkoh frames and the second CDS raster as background to be subtracted pixel-bypixel from the other frames. The advantages and disadvantages of this method of background subtraction are listed in Sect. 2; a simple inspection of the background-subtracted images supports that the method provides sound results. The background is indeed a significant fraction $(>50 \%)$ of the total signal for all 
instruments (Fig. 5), and its accurate subtraction is therefore critical for any subsequent analysis and for determining any physical parameter of a specific structure. We find differences between indicators obtained from subtracted and unsubtracted data (Fig. 8), the former being less uniform and more evolving.

\subsection{Loop morphology and evolution}

The loop is best visible and lives longer in the TRACE $171 \AA$ and $195 \AA$ filter passbands. It is instead quite faint and decays rapidly in the $284 \AA$ filter passband.

A simple superposition of the images, using the loop outlines, shows that the loop in the $171 \AA$ filter passband reasonably overlaps the loop in the $195 \AA$ filter passband. The good correspondence is also supported by the loop aspect in the $195 / 171$ filter ratio maps. The alignment is not as good with the loop in the $284 \AA$ filter passband. The SXT loop is more diffuse: the right leg appears to overlap that of the TRACE $171 \AA$ / $/ 195 \AA$ loop. This may indicate the presence of relatively hot plasma in the right leg of the loop. The emission evolution in the SXT band appears to be quite coherent with that in the TRACE $195 \AA$ band. However, because of the lower spatial resolution, no conclusive statement can be made on the correspondence between the SXT and the TRACE loop. With the same limitations, the TRACE loop overlaps well with the loop as visible in a few SoHO/CDS spectral lines. The loop is best visible in the Mg IX $368 \AA$ line, but well visible also in the Mg X $625 \AA$, in the Si X $347 \AA$ and $356 \AA$ lines, less in the Fe XII $364 \AA$ line. All these lines have temperatures of maximum formation about $\log T \sim 6.0-6.1$, which is also around the temperature of maximum sensitivity of both the TRACE $171 \AA$ and $195 \AA$ filter passbands. Thus, there is a qualitative coherence of the data from the different instruments. Limited parts of the loop seem to be visible in a few spectral lines with other formation temperatures, e.g. the left leg in the cooler $\mathrm{Ca} X$ line $(\log T \approx 5.9)$, the lower part of the right leg in the hotter Fe lines. This suggests that, during the first CDS raster, in the right leg there is hotter plasma than in the left leg. This is confirmed by the $\operatorname{EM}(\mathrm{T})$ reconstructed at five locations along the loop, after removing the $\mathrm{Mg}$ X $625 \AA$ line. In the cool O V line, we may be seeing only the footpoints of the loop.

We will not comment here about the structures surrounding the selected loop. There is another bright structure that apparently intersects the loop and that may have importance because it appears to evolve coherently with the loop. This may be taken as evidence of an interaction of this structure with the loop.

The TRACE images show that the loop is substructured in several strands, as are many other loops observed with TRACE. This work analyzes the loop as a single and coherent structure, and we will not comment further on the substructuring of the loop, which may deserve a further separate work. We only note that TRACE appears to be detecting a thermal structuring across the loop better than other instruments, as shown by the filter ratio maps. Since the loop appears to have a coherent evolution, the finer substructuring may not be crucial for the
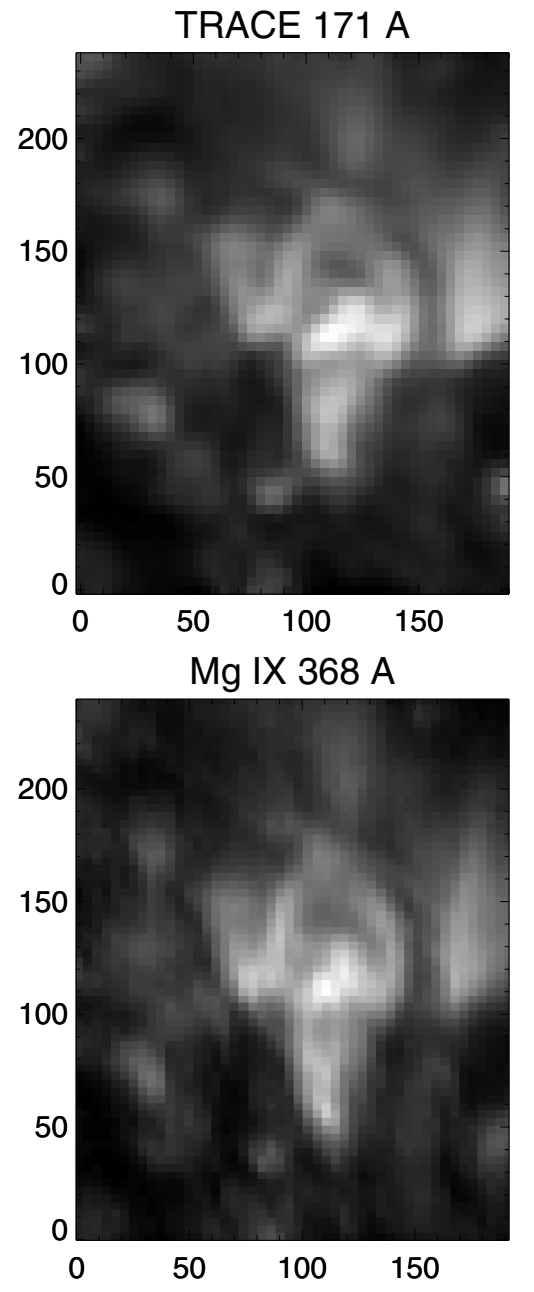

Fig. 13. Image of the loop region obtained from the TRACE $171 \AA$ images with a procedure which mimics the CDS rastering (at corresponding times) and degraded to the CDS angular resolution (with a further smoothing with a $2 \times 2$ pixel boxcar), compared to the CDS (first) raster image in the Mg IX $368 \AA$ line. The image coordinates are in arcsec.

description of average properties of the system. The coherent evolution may suggest a coherent heating across the structure.

TRACE and Yohkoh data allow us to analyze the evolution of the loop. Unfortunately, CDS data are not able to provide analogous information because there is only one relevant raster, i.e. a single snapshot, during the loop evolution. High quality spectroheliograms intrinsically require relatively long acquisition times.

In the analyzed time sequence the loop evolves. The TRACE $171 \AA$ filter detects an evolution both of the emission intensity and of its distribution along the loop. In particular, the right leg of the loop is bright at the beginning of the observation, while the left leg becomes the brighter later on. The bright left leg is coherent with the brightness distribution in the relevant CDS lines, taken in the same time period. Figure 13 shows an image of the loop region obtained from the TRACE $171 \AA$ images with a procedure that mimics the CDS rastering (with corresponding time stepping) and degraded to the CDS angular resolution, compared to the CDS raster 
image in the Mg IX $368 \AA$ line. The figure confirms the very good correspondence of the loop appearance in both images. The asymmetry along the loop both in space and in time may be an indication of an asymmetric distribution of the heating along the loop and a time variation of the heating intensity, to be checked through detailed modeling. In the SXT and the other TRACE filters the emission distribution is more uniform and evolves more uniformly.

The SoHO campaign covers mostly the loop decay. This is clear from the relevant light curves (shown in Fig. 6): they have a decreasing trend, with e-folding times between 0.7 and $1.7 \mathrm{~h}$, with the only exception of the TRACE $171 \AA$ filter. In this filter, the light curve has a peak about one hour after the observation starts and then decays steadily. This is coherent with other TRACE observations of loops (Winebarger et al. 2003b). A coherent interpretation of the light curves is compatible with a progressive cooling of the loop: the intensity drops earlier in the filter passbands more sensitive to higher temperatures (SXT, TRACE $284 \AA$ ). The peak in the $171 \AA$ filter passband, which is sensitive to cooler plasma than the others, may be simply due to the progressive entrance of the cooling loop plasma into the temperature regime of the highest filter sensitivity. We have also checked that the loop had been bright and relatively steady for quite a long time (about four hours) before the start of the campaign.

The TRACE light curve decay times $(\sim 1 \mathrm{~h})$ are significantly longer that the characteristic cooling time expected for a loop of this length from the equilibrium $(\sim 1 / 2 \mathrm{~h}$ or shorter). This may suggest that the loop decay is sustained by a slowly decaying heating, as often occurs in flaring loops (e.g. Sylwester et al. 1993). We cannot exclude other possible interpretations, e.g. an envelope of the free cooling of different loop strands ignited at delay one from the other (Warren et al. 2003) and/or significantly lower metal abundances. Detailed loop modeling may shed further light on this topic.

\subsection{Loop physical conditions}

The pixel-by-pixel background subtraction allows us to derive TRACE 195/171 filter ratio maps. The maps show a moderate structuring of the filter ratio across the loop, with relatively higher filter ratio values in the inner shell and at the apex of the loop (Fig. 7). The filter ratio maps also show that the filter ratio has a coherent structure along the loop and that it evolves. This can be seen also in the background-subtracted filter ratio profiles along the loop. The right leg of the loop does not intersect with other bright structures along the line of sight and the filter ratio diagnostics seem to be more reliable there. We see a filter ratio peak there in the initial profiles, which may be the signature of a temperature maximum. In the left leg the profiles show a deep minimum, which may be taken as a signature of a monotonic decrease of the temperature toward the left footpoint. The evidence is not conclusive, because of the presence of a bright co-evolving structure which intersects along the line of sight. The filter ratio profiles along the loop are moderately non-uniform. Other transient ratio peaks sometimes occur mostly at the right visible footpoint. Although cautiously, we suggest that these peaks may be the signature of minor heating episodes.

The 195/171 ratio values are mostly contained in the range $0.1-5$, which should correspond to a temperature range $6.0 \leq \log T \leq 6.2$. Initially, the higher ratio values and the good visibility in the TRACE $195 \AA$ A band indicate the presence of relatively hotter plasma than later, with better evidence in the right leg of the loop. The right leg of the loop is also relatively bright at early times in the $284 \AA$ band and in the SXT band, suggesting the presence of even hotter plasma in this phase and, therefore, a moderately multi-thermal structure across the loop. The absence of spectral data in this phase does not allow us to confirm this indication. The SXT and TRACE $284 \AA$ light curves show however that such hotter plasma rapidly cools down and, coherently, is no longer present during the CDS raster. We may conjecture the presence of hot strands at the beginning of the campaign which either decay more rapidly than the others or become uniform to them; this may mean that in the life of the same loop we may find more and fewer multi-thermal phases at different times. This may not be in disagreement with the presence of both multi-thermal and isothermal cross-structures suggested by Schmelz et al. (2005) and represent a further piece in the puzzle. Detailed modeling, and, more probably, next generation multi-band imaging observations will help to clarify this important issue.

Later, during the first CDS raster, the loop probably cools closer to $\log T \approx 6.0-6.1$. This temperature regime is largely coherent with the loop appearance in the spectral lines of SoHO/CDS: the loop is best visible in the Mg IX $368 \AA$ line, which peaks at $\log T \approx 6.0$, and we see the loop also in the Mg X $625 \AA$, Fe XII $364 \AA$, and in the Si X lines $(\log T \approx 6.1)$. The loop is instead barely visible in the lines just outside this temperature regime. By combining CDS data with theoretical line emissivities, we can sample emission measure distributions along the loop. After removing the Mg X $625 \AA$ line, clearly inconsistent with all others, we obtain that the hottest components lie on the right side of the loop (CDS sector 9), with a peak temperature of $\log T \approx 6.25$ (Fig. 12). The emission measure drops sharply above this temperature, and generally more smoothly to the cool side. At this time, on the right leg, the TRACE 195/171 filter ratio is around 1, corresponding to a temperature $\log T \approx 6.1$. This may suggest that, in this moderately multi-thermal structure, TRACE is more sensitive to the plasma at intermediate temperature. It will be interesting to check this hypothesis through detailed loop forward modeling. Assuming a loop thickness $\sim 5 \times 10^{8} \mathrm{~cm}$ an emission measure of $\sim 10^{27} \mathrm{~cm}^{-5}$ is compatible with a density $\sim 1.5 \times 10^{9} \mathrm{~cm}^{-3}$, moderately overdense with respect to a loop at temperature $\log T \approx 6.2$ (according to the scaling laws of Rosner et al. 1978), consistent with the fact that the loop is cooling.

The TRACE filter ratio values averaged along the loop (Fig. 8) definitely decrease with time, supporting the progressive cooling of the loop. The decrease somewhat slows with time, perhaps indicating the occurrence of secondary heating episodes, which would be consistent with the observed long decay time of the loop. 


\section{Conclusions}

In this work we use a combination of multi-wavelength data, time/space/spectral resolution and signal-to-noise ratio to investigate the possible extent of direct diagnostics and interpretation of a coronal observation. The observation of a specific structure, a coronal loop, and of its evolution helps us find coherent results and limitations to the information that it is possible to derive.

The analysis of the collected information shows that it is possible to obtain a coherent scenario and to detect several details of the emission and evolution of a coronal loop. It confirms that temperature diagnostics with TRACE are difficult, and a proper subtraction of the high background is critical, but also indicates that, in particular conditions, some sound information can be obtained. Spectroscopic data from SoHO/CDS provide useful complementary information, constraining the loop thermal structure, although in the limit of lower temporal and spatial resolution.

The coherent scenario that we obtain across bands and instruments appears to confirm the overall evolution of the coronal loops entirely visible with TRACE (Warren et al. 2002, 2003) and the presence of thermal structuring (Schmelz et al. 2005), but also adds several qualitative and quantitative details and puts several constraints to be matched coherently through detailed loop modeling.

Acknowledgements. We thank J. C. Raymond, H. Hudson, J. Klimchuk and A. Maggio for suggestions. The authors acknowledge support for this work from Agenzia Spaziale Italiana and Ministero dell'Istruzione, Università e Ricerca.

\section{References}

Aschwanden, M. J. 2001, ApJ, 559, L171

Aschwanden, M. J. 2002, ApJ, 580, L79

Aschwanden, M. J., \& Nightingale, R. 2005, ApJ, in press

Aschwanden, M. J., Schrijver, C. J., \& Alexander, D. 2001, ApJ, 550, 1036

Cargill, P. J., \& Klimchuk, J. A. 2004, ApJ, 605, 911

De Moortel, I., Ireland, J., \& Walsh, R. W. 2000, A\&A, 355, L23

Domingo, V., Fleck, B., \& Poland, A. I. 1995, Sol. Phys., 162, 1

Handy, B. N., Acton, L. W., Kankelborg, C. C., et al. 1999, Sol. Phys., 187,229

Harrison, R. A., Sawyer, E. C., Carter, M. K., et al. 1995, Sol. Phys., 162,233

Landi, E., Feldman, U., \& Dere, K. P. 2002a, ApJS, 139, 281

Landi, E., Feldman, U., \& Dere, K. P. 2002b, ApJ, 574, 495
Landi, E., \& Feldman, U. 2004, ApJ, 611, 537

Landi, E., \& Landini, M. 2004, ApJ, 608, 1133

Lanzafame, A. C., Brooks, D. H., \& Lang, J. 2005, A\&A, 432, 1063

Lenz, D. D., Deluca, E. E., Golub, L., Rosner, R., \& Bookbinder, J. A. 1999, ApJ, 517, L155

Martens, P. C. H., Cirtain, J. W., \& Schmelz, J. T. 2002, ApJ, 577, L115

Mazzotta, P., Mazzitelli, G., Colafrancesco, S., \& Vittorio, N. 1998, A\&AS, 133, 403

Nagata, S., Hara, H., Kano, R., et al. 2003, ApJ, 590, 1095

Nakariakov, V. M., \& Ofman, L. 2001, A\&A, 372, L53

Ogawara, Y., Takano, T., Kato, T., et al. 1991, Sol. Phys., 136, 1

Patsourakos, S., Klimchuk, J. A., \& MacNeice, P. J. 2004, ApJ, 603, 322

Priest, E. R., Foley, C. R., Heyvaerts, J., et al. 2000, ApJ, 539, 1002

Reale, F. 2002, ApJ, 580, 566

Reale, F., \& Peres, G. 2000, ApJ, 528, L45

Reale, F., Peres, G., Serio, S., et al. 2000, ApJ, 535, 423

Rosner, R., Tucker, W., \& Vaiana, G. 1978, ApJ, 220, 643

Schmelz, J. T. 2002, ApJ, 578, L161

Schmelz, J. T., Beene, J. E., Nasraoui, K., et al. 2003, ApJ, 599, 604

Schmelz, J. T., Nasraoui, K., Richardson, V. L., et al. 2005, ApJ, 627, L81

Schmelz, J. T., Scopes, R. T., Cirtain, J. W., Winter, H. D., \& Allen, J. D. 2001, ApJ, 556, 896

Schmieder, B., Rust, D. M., Georgoulis, M. K., Démoulin, P., \& Bernasconi, P. N. 2004, ApJ, 601, 530

Schrijver, C. J., et al. 1999, Sol. Phys., 187, 261

Serio, S., Peres, G., Vaiana, G. S., Golub, L., \& Rosner, R. 1981, ApJ, 243, 288

Serio, S., Reale, F., Jakimiec, J., Sylwester, B., \& Sylwester, J. 1991, A\&A, 241, 197

Sylwester, B., Sylwester, J., Serio, S., et al. 1993, A\&A, 267, 586

Testa, P., Peres, G., Reale, F., \& Orlando, S. 2002, ApJ, 580, 1159

Testa, P., Peres, G., \& Reale, F. 2005, ApJ, 622, 695

Tsuneta, S., Acton, L., Bruner, M., et al. 1991, Sol. Phys., 136, 37

Vaiana, G. S., Krieger, A. S., \& Timothy, A. F. 1973, Sol. Phys., 32, 81

Warren, H. P., \& Reeves, K. K. 2001, ApJ, 554, L103

Warren, H. P., Winebarger, A. R., \& Hamilton, P. S. 2002, ApJ, 579, L41

Warren, H. P., Winebarger, A. R., \& Mariska, J. T. 2003, ApJ, 593, 1174

Winebarger, A. R., DeLuca, E. E., \& Golub, L. 2001, ApJ, 553, L81

Winebarger, A. R., Warren, H., van Ballegooijen, A., DeLuca, E. E., \& Golub, L. 2002, ApJ, 567, L89

Winebarger, A. R., Warren, H. P., \& Mariska, J. T. 2003a, ApJ, 587, 439

Winebarger, A. R., Warren, H. P., \& Seaton, D. B. 2003b, ApJ, 593, 1164 